\title{
Erratum to: Transactions on Large-Scale Data- and Knowledge-Centered Systems XXXVI
}

\author{
Abdelkader Hameurlain ${ }^{1(\bowtie)}$, Josef Küng ${ }^{2}$, Roland Wagner ${ }^{2}$, \\ Tran Khanh Dang ${ }^{3}$, and Nam Thoai ${ }^{3}$ \\ ${ }^{1}$ IRIT, Paul Sabatier University, Toulouse, France \\ hameur@irit.fr \\ ${ }^{2}$ FAW, University of Linz, Linz, Austria \\ ${ }^{3}$ Ho Chi Minh City University of Technology, Ho Chi Minh City, Vietnam
}

\section{Erratum to:}

\section{A. Hameurlain et al. (Eds.): \\ Transactions on Large-Scale Data- and Knowledge-Centered Systems XXXVI, LNCS 10720, https://doi.org/10.1007/978-3-662-56266-6}

The subtitle "Special Issue on Data and Security Engineering" was omitted from an earlier version of the cover and frontmatter pages of this publication. 